\title{
TITLE:
}

\section{Intracranial arachnoid cysts in a chimpanzee (Pan troglodytes).}

\section{AUTHOR(S):}

Miyabe-Nishiwaki, Takako; Kaneko, Takaaki; Sakai, Tomoko; Kaneko, Akihisa; Watanabe, Akino; Watanabe, Shohei; Maeda, Norihiko; ... Tomonaga, Masaki; Matsuzawa, Tetsuro; Mikami, Akichika

\section{CITATION:}

Miyabe-Nishiwaki, Takako ... [et al]. Intracranial arachnoid cysts in a chimpanzee (Pan troglodytes).. Primates 2013, 55(1): 7-12

\section{ISSUE DATE:}

2013-09-26

URL:

http://hdl.handle.net/2433/197658

\section{RIGHT:}

The final publication is available at Springer via http://dx.doi.org/10.1007/s10329-0130384-0; この論文は著者最終稿です。内容が印刷版と異なることがありますので、引用 の際には出版社版をご確認ご利用ください。This is the Accepted Author Manuscript. Please cite only the published version. 
3 Takako Miyabe-Nishiwaki ${ }^{1}$, Takaaki Kaneko ${ }^{1}$, Tomoko Sakai ${ }^{1}$, Akihisa Kaneko ${ }^{1}$, Akino

4 Watanabe $^{1}$, Shohei Watanabe ${ }^{1}$, Norihiko Maeda ${ }^{1}$, Kiyonori Kumazaki ${ }^{1}$, Juri Suzuki ${ }^{1, *}$,

$5 \quad$ Reina Fujiwara ${ }^{2}$, Haruyuki Makishima ${ }^{3}$, Takeshi Nishimura ${ }^{1}$, Misato Hayashi ${ }^{1}$, Masaki

6 Tomonaga $^{1}$, Tetsuro Matsuzawa ${ }^{1}$, Akichika Mikami ${ }^{4}$

7

$8{ }^{1}$ Primate Research Institute, Kyoto University, Inuyama, Aichi, Japan

$9 \quad{ }^{2}$ Veterinary Medical Center, The University of Tokyo, Tokyo, Japan

$10{ }^{3}$ Laboratory of physical anthropology, Kyoto University, Kyoto, Japan

$11{ }^{4}$ Chubu Gakuin University, Gifu, Japan

12

13 Correspondence: Dr. Juri Suzuki

14 Primate Research Institute, Kyoto University

15 41-2 Kanrin, Inuyama, Aichi 484-8506, Japan

16 Tel: +81-568-63-0586 Email: suzuki.juri.4u@kyoto-u.ac.jp (J. Suzuki) 


\section{Abstract}

19 An intracranial arachnoid cyst was detected in a 32-year-old, 44.6-kg, female

20 chimpanzee at the Primate Research Institute, Kyoto University. Magnetic resonance imaging (MRI) and computed tomography (CT) were performed and the cognitive studies in which she participated were reviewed. MRI revealed that the cyst was present in the chimpanzee's right occipital convexity, and was located in close proximity to the posterior horn of the right lateral ventricle without ventriculomegaly. CT confirmed the presence of the cyst and no apparent signs indicating previous skull fractures were found. The thickness of the mandible was asymmetrical, whereas the temporomandibular joints and dentition were symmetrical. She showed no abnormalities in various cognitive studies since she was 3 years old, except a different behavioural pattern during a recent study, indicating a possible visual field defect. Detailed cognitive studies, long-term observation of her physical condition and follow-up MRI will be continued. 


\section{Introduction}

An arachnoid cyst is an accumulation of intra-arachnoid fluid, which can be congenital or secondary to inflammation, brain trauma, haemorrhage, chemical irritation or tumours (Cincu et al. 2007). In humans, most arachnoid cysts are detected during the first two decades of life (Gosalakkal 2002). They are often asymptomatic and are encountered as incidental findings of neuroimaging (Spansdahl and Solheim 2007). Arachnoid cysts can cause headaches, seizures, craniomegaly, developmental delays and, rarely, hemiparesis as well as various defects specific to the location of the cysts. The aetiology of congenital cysts remains unclear, but they are considered to be developmental anomalies of the arachnoid membrane, which resulted in the accumulation of cerebrospinal fluid (CSF)-like fluid. Arachnoid cysts are mainly supratentorial (90\%) and are occasionally present in the posterior fossa (10\%). The most common supratentorial site is the middle cranial fossa (60\%) and other sites include the quadrigeminal plate, sellar region and convexity (Cincu et al. 2007).

Intracranial arachnoid cysts have also been reported in cattle (Lee et al. 2009) and dogs (Vernau et al. 1997; Kitagawa et al. 2003; Dewey et al. 2007; Wyss-Fluehmann et al. 2008), whereas spinal arachnoid cysts are more common in dogs and cats (Skeen et al. 2003). However, arachnoid cysts in non-human primates have rarely been documented. In the present report, we describe magnetic resonance imaging (MRI) and computed tomography (CT) findings in a female chimpanzee with an intracranial arachnoid cyst and briefly review the cognitive studies in which she participated.

\section{Case Report}

An intracranial arachnoid cyst was detected in a 32-year-old, 44.6-kg (at the time of 
initial MRI), female chimpanzee named Pendesa at the Primate Research Institute, Kyoto University (KUPRI). The chimpanzee was kept in an indoor-outdoor enclosure in a social group comprising 2 males and 4 females: see (Matsuzawa 2003, 2006) for further information concerning the social group.

The chimpanzee was born at the Japan Monkey Centre (JMC) in 1977 and was transferred to KUPRI in 1979 when she was two years old. She participated in various cognitive research studies since then, but has never been used for medical research. All studies after 1986 were conducted under the guidelines provided by the KUPRI after the approval of institutional Animal Welfare and Care Committee. She was diagnosed with atopic dermatitis in 2000, which has been controlled with an oral antihistamine (Salatine, Nipro Genepha Corporation, Saitama, Japan) and topical application of jojoba oil during the dry seasons. No neurological signs were noted before MRI (General Electrics Signa Profile MRI scanner, 0.2 T, GE Yokoo Medical Systems Co., Tokyo, Japan) was performed for research purposes.

\section{Results}

The chimpanzee was anesthetized with ketamine hydrochloride $(100 \mathrm{mg} / \mathrm{mL}$ Ketalar ${ }^{\circledR}, 3.5$ mg/kg; Sankyo-Parke-Davis \& Co., Inc., Japan) and medetomidine hydrochloride (0.035 mg/kg; Domitor ${ }^{\circledR}$; Meiji Seika Kaisha, Ltd., Tokyo, Japan). Anaesthesia was maintained with isoflurane (Isoflu; Dainippon Sumitomo Pharma Co., Ltd. , Osaka, Japan) delivered in oxygen through a precision vaporizer and a rebreathing circuit. MRI was performed to study the morphology of chimpanzee’s brain (Sakai et al. 2011), which revealed an arachnoid cyst in the right occipital convexity (Fig. 1A-C). The cyst was located in close proximity to and possibly communicated with the posterior horn of the right lateral ventricle, but no evidence of ventriculomegaly was 
observed. T1- and T2-weighted MRI signals in the cyst were similar to those in CSF. These images were viewed and discussed with both veterinary and human neurologists. We acquired three-dimensional (3D) T1-weighted whole brain images using the 3D fast gradient echo imaging sequence. The images were analysed using the following series of manual and automated procedures: (i) analyses using Analyze 9.0 software (Mayo Clinic, Rochester, MN, USA) and conversion to cubic voxel dimensions of 0.55 mm using a cubic spline interpolation algorithm, (ii) re-alignment of brain image volumes to a standard anatomical orientation with the transaxial plane parallel to the anterior commissure-posterior commissure line and perpendicular to the interhemispheric fissure, (iii) manual tracing and measurement of the entire arachnoid cyst by one of the image analysts (T.S.) in consultation with a veterinarian (J.S.) and (iv) calculation of the absolute volume of the arachnoid cyst from an automatic count of the number of voxels per $\mathrm{cm}^{3}$ using Analyze 9.0 software, which resulted in a total volume of $2.8 \mathrm{~cm}^{3}$.

One year after the initial MRI, the chimpanzee was anesthetized as mentioned above except that sevoflurane (Sevoflo; Dainippon Sumitomo Pharma Co., Ltd. , Osaka, Japan) was used instead of isoflurane and CT was performed using the Asteion CT scanner (model no. TSX-021B; Toshiba Medical Systems Corporation, Tochigi, Japan), which revealed an arachnoid cyst that did not appear to change in size over the preceding one year. The chimpanzee's skull was smooth, and CT did not reveal apparent signs indicating previous skull fractures. However, the thickness of her mandible was asymmetrical, whereas her temporomandibular joints and dentition were symmetrical (Fig. 1D). These images were viewed and discussed with a human dentist. She has not shown any developmental delays or other behavioural 
111 abnormalities, but researchers and her caretakers have noticed that she frequently

112 rocked back and forth while sitting.

113 Table 1 lists the cognitive studies in which Pendesa participated since she was

1143 years old. She participated in various cognitive tests using visual and auditory

115 modalities, but showed no inferiority to the other chimpanzees in any respect, except in

116 a colour classification task (Matsuno et al. 2004). She performed the colour

117 classification task when she was 23 years old and showed less stable classification

118 compared with a female chimpanzee named Ai (Matsuno et al. 2004, review in Matsuno

119 et al. 2006). Ai and Pendesa were the same age and both reared by human. Although

120 they had similar history, only Ai had learned symbolic colour names through long-term

121 training. In this task, Matsuno and colleagues adopted a "nonlinguistic" test to directly

122 compare colour classification by these two chimpanzees. They were shown 124 test

123 colours and asked to match to 9 standard colours, not to symbols. As a result, Pendesa

124 showed significantly less consistent classifications.

125 The results of the recent cognitive study (conducted when she was 33 years

126 old) indicate that Pendesa had different behavioural patterns, suggesting a possible

127 defect in her left visual field (Kaneko et al., under review). In this study, the

128 detectability of the small light spot presented on several locations of visual field was

129 measured while monitoring the gaze positions by infra-red remote eye-tracker. As a

130 result, the detectability was close to zero around the bottom-left quadrant of visual field.

\section{Discussion}

An arachnoid cyst was detected in the right occipital convexity in a clinically

133 healthy, adult, female chimpanzee during MRI for research purposes. One year later, CT confirmed the presence of the cyst and asymmetrical thickness of the chimpanzee's 
mandible. Although it is difficult to differentiate arachnoid cysts and epidermoid cysts or dermoid cysts (ie., if the content of the cyst was cerebrospinal fluid or something else) without diffusion weighted images, in the present case, arachnoid cyst was the most consistent with our MRI and CT images (shape, size and the location of the cyst), history and symptoms.

In humans, occipital convexity arachnoid cysts are rare, but two case reports have documented symptomatic arachnoid cysts in elderly women (Tucker et al. 2006; Suzuki et al. 2009). The cyst volume slowly increased over time in one case; the cyst was located close to the patient's posterior horn of the right lateral ventricle, which may

144 have been related to the cystic growth (Suzuki et al. 2009). The other woman with a cystic lesion in the right occipital convexity presented with a visual field defect and headache. A visual field examination showed left lower quadrantanopia. Surgical treatment was performed and her headache and visual field defect improved (Suzuki et al. 2009).

In the present case, an arachnoid cyst was located in the right occipital convexity, which was considered to be mostly in V1 area (Bailey et al. 1950). Neither the researchers nor caretakers noticed any developmental delays or other behavioural

152 abnormalities until recently except frequent to-and-fro rocking while sitting. The chimpanzee has participated in various cognitive studies measuring a wide range of

154 visual and auditory functions without any intervals since she was 3 years old. She

155 showed no abnormalities or inferiority in performance and was occasionally even better

156 than other chimpanzees in various tasks, except for the colour classification task

157 (Matsuno et al 2004). Matsuno and colleagues interpreted that Pendesa classified colours less stably because she had less training and limited understanding of colour 
names. It was unlikely that her colour classification ability was affected by the presence

of the arachnoid cyst. More recently, the left visual field defect was suggested in a

cognitive study (Kaneko et al, under review). This suspected left quadrantanopia was consistent with a defect that was predicted from the cyst location.

trauma (Gosalakkal 2002; Cincu et al. 2007). In the present case, a history of brain

trauma was not recorded after the chimpanzee was transferred to KUPRI at the age of 2

skull fractures were not found, but CT revealed obvious mandibular asymmetry. The

causes of mandibular asymmetry can be developmental, traumatic, pathological (e.g., tumour, cysts, infection) or functional (mandibular displacement) (Chia et al 2008). In this case, traumatic, pathological and functional causes were not found and it appears to be similar to the developmental condition, hemimandibular hyperplasia in humans (Chia et al 2008). The asymmetry was not obvious from photographs

173 (http://langint.pri.kyoto-u.ac.jp/ai/en/friends/pendesa.html) and it was not clear when the condition developed in her life. Reportedly, patients with congenital arachnoid cysts occasionally have additional malformations (Cincu et al. 2007). Collectively, it is possible that the arachnoid cyst and the mandibular asymmetry were both based on her genetic background. However, if a suspected visual field defect is, in fact, associated with a cyst, it can be contradictory to a congenital cyst because the function is likely to

179 be compensated during development. In such cases, the cyst might have developed at some point after birth and gradually expanded to eventually show symptoms. A

181 histopathological examination can distinguish congenital cysts from secondary cysts because the walls in congenital cysts contain arachnoid cells connected with unchanged 
arachnoid matter, whereas those in secondary cysts contain arachnoid scarring (Cincu et al. 2007).

It was difficult to assess whether the chimpanzee had headaches. At least, she did not seem to suffer from headaches to the extent that her social life was impaired. Her frequent rocking behaviour was considered to be a stereotype behaviour and was caused by stress during tasks and/or social influences, despite the enriched environment (Matsuzawa 2003, 2006). However, if she did have a left visual field defect, it could be speculated that she was actually using motion parallax to compensate for her impaired visual field.

We believe that this is the first description of an arachnoid cyst causing possible visual defects in a chimpanzee. Precise behavioural testing on visual fields and blindness is in progress. The general behaviour and physical condition of Pendesa will be continuously observed and follow-up MRI will be performed throughout her lifetime to determine the course of the cyst.

\section{Acknowledgments}

We wish to thank Prof. Kozo Matsubayashi at the Center for Southeast Asian Studies, Kyoto University for his assistance in interpreting the MRI images and Dr. W. Saito at Tsurumi University for his assistance in interpreting the CT images. We thank Prof. S. Hirata at Wildlife Research Centre, Kyoto University, Dr. M. Tanaka at Wildlife Research and Education Center, Kyoto City Zoo, and Dr. S. Itakura at Department of Psychology, Kyoto University for valuable information.

This study was financially supported by the following grants: MEXT 16002001, 20002001, 24000001 (to T.M.) and JSPS-gCOE (A06). 


\section{References}

208

Anderson JR, Myowa-Yamakoshi M, Matsuzawa T (2004) Contagious yawning in chimpanzees. Proceedings Biological sciences / The Royal Society 271 Suppl 6:S468-470. doi:10.1098/rsbl.2004.0224

Bailey P, Bonin GV, McCulloch WS (1950) The isocortex of the chimpanzee. University of Illinois Press, Urbana

Chia MSY, Naini FB, Gill DS (2008) The aetiology, diagnosis and management of mandibular asymmetry. Orthodontic Update 2008; 1:44-52

Cincu R, Agrawal A, Eiras J (2007) Intracranial arachnoid cysts: current concepts and treatment alternatives. Clinical neurology and neurosurgery 109 (10):837-843. doi:10.1016/j.clineuro.2007.07.013

Dewey CW, Krotscheck U, Bailey KS, Marino DJ (2007) Craniotomy with cystoperitoneal shunting for treatment of intracranial arachnoid cysts in dogs. Veterinary surgery : VS 36 (5):416-422. doi:10.1111/j.1532-950X.2007.00287.x

Gosalakkal JA (2002) Intracranial arachnoid cysts in children: a review of pathogenesis, clinical features, and management. Pediatric neurology 26 (2):93-98

Goto K, Imura T, Tomonaga M (2012) Perception of emergent configurations in humans (Homo sapiens) and chimpanzees (Pan troglodytes). Journal of experimental psychology Animal behavior processes 38 (2):125-138. doi:10.1037/a0026899

Hirata S, Matsuzawa T (2001) Tactics to obtain a hidden food item in chimpanzee pairs (Pan troglodytes). Animal cognition 4 (3-4):285-295. doi:10.1007/s100710100096

Hirata S (2007) A note on the responses of chimpanzees (Pan troglodytes) to live self-images on television monitors. Behavioural processes 75 (1):85-90. doi:10.1016/j.beproc.2007.01.005 
Huffman MA, Hirata S (2004) An experimental study of leaf swallowing in captive chimpanzees: insights into the origin of a self-medicative behavior and the role of social learning. Primates; journal of primatology 45 (2):113-118. doi:10.1007/s10329-003-0065-5

Imura T, Tomonaga M, Yagi A (2008) The effects of linear perspective on relative size discrimination in chimpanzees (Pan troglodytes) and humans (Homo sapiens). Behavioural processes 77 (3):306-312. doi:10.1016/j.beproc.2007.07.006

Itakura S, Tanaka M (1998) Use of experimenter-given cues during object-choice tasks by chimpanzees (Pan troglodytes), an orangutan (Pongo pygmaeus), and human infants (Homo sapiens). Journal of comparative psychology 112 (2):119-126

Iversen IH, Matsuzawa T (1996) Visually guided drawing in the chimpanzee (Pan troglodytes)1. Japanese Psychological Research 38 (3):126-135. doi:10.1111/j.1468-5884.1996.tb00017.x

Iversen IH, Matsuzawa T (1997) Model-guided Line Drawing in the Chimpanzee (Pan troglodytes). Japanese Psychological Research 39 (3):154-181. doi:10.1111/1468-5884.00051

Iversen IH, Matsuzawa T (2003) Development of interception of moving targets by chimpanzees (Pan troglodytes) in an automated task. Animal cognition 6 (3):169-183. doi:10.1007/s10071-003-0175-x

Kaneko T, Tomonaga M (2011) The perception of self-agency in chimpanzees (Pan troglodytes). Proceedings Biological sciences / The Royal Society 278 (1725):3694-3702. doi:10.1098/rspb.2011.0611

Kaneko T, Tomonaga M (2012) Relative contributions of goal representation and kinematic information to self-monitoring by chimpanzees and humans. Cognition 125 
Kitagawa M, Kanayama K, Sakai T (2003) Quadrigeminal cisterna arachnoid cyst diagnosed by MRI in five dogs. Australian veterinary journal 81 (6):340-343

Kojima S, Kiritani S (1989) Vocal-auditory functions in the chimpanzee: Vowel perception.

Kojima S, Tatsumi IF, Kiritani S, Hirose H (1989) Vocal-auditory functions of the chimpanzee: consonant perception. Hum Evol 4 (5):403-416. doi:10.1007/BF02436436

Kojima S (1990) Comparison of auditory functions in the chimpanzee and human. Folia primatologica; international journal of primatology $55(2): 62-72$

Lee K, Yamada K, Tsuneda R, Kishimoto M, Shimizu J, Kobayashi Y, Furuoka H, Matsui T, Sasaki N, Ishii M, Inokuma H, Iwasaki T, Miyake Y (2009) Clinical experience of using multidetector-row CT for the diagnosis of disorders in cattle. The Veterinary record 165 (19):559-562

Matsuno T, Kawai N, Matsuzawa T (2004) Color classification by chimpanzees (Pan troglodytes) in a matching-to-sample task. Behavioural brain research 148

Matsuno T, Kawai N, Matsuzawa T (2006) Color Recognition in Chimpanzees (Pan troglodytes) In: Matsuzawa T (ed) Cognitive Development in Chimpanzees. . Springer-Verlag, Tokyo, Japan, pp 317-329

Matsuno T, Tomonaga M (2006) Visual search for moving and stationary items in chimpanzees (Pan troglodytes) and humans (Homo sapiens). Behavioural brain research 172 (2):219-232. doi:10.1016/j.bbr.2006.05.004 
chimpanzees (Pan troglodytes). Behavioural processes 75 (3):253-258.

280 doi:10.1016/j.beproc.2007.02.028

281

Matsuno T, Tomonaga M (2008) Temporal characteristics of visibility in chimpanzees (Pan troglodytes) and humans (Homo sapiens) assessed by a visual-masking paradigm. Perception 37 (8):1258-1268

Matsuzawa T (2003) The Ai project: historical and ecological contexts. Animal cognition 6 (4):199-211. doi:10.1007/s10071-003-0199-2

Matsuzawa T (2006) Sociocognitive development in chimpanzees: a synthesis of laboratory work and field work. I. In: Matsuzawa T (ed) Cognitive Development in Chimpanzees. . Springer-Verlag, Tokyo, Japan, pp 3-33

Sakai T, Mikami A, Tomonaga M, Matsui M, Suzuki J, Hamada Y, Tanaka M, Miyabe-Nishiwaki T, Makishima H, Nakatsukasa M, Matsuzawa T (2011) Differential prefrontal white matter development in chimpanzees and humans. Current biology : CB 21 (16):1397-1402. doi:10.1016/j.cub.2011.07.019

Skeen TM, Olby NJ, Munana KR, Sharp NJ (2003) Spinal arachnoid cysts in 17 dogs. Journal of the American Animal Hospital Association 39 (3):271-282

Sousa C, Matsuzawa T (2001) The use of tokens as rewards and tools by chimpanzees (Pan troglodytes). Animal cognition 4 (3-4):213-221. doi:10.1007/s100710100104

Spansdahl T, Solheim O (2007) Quality of life in adult patients with primary intracranial arachnoid cysts. Acta neurochirurgica 149 (10):1025-1032; discussion 1032. doi:10.1007/s00701-007-1272-4

Suzuki M, Tamaki T, Toda S, Tsuchiya M, Kogure K, Hosone M, Node Y, Teramoto A (2009) Delayed recurrent arachnoid cyst of the occipital convexity in an elderly woman. Neurologia medico-chirurgica 49 (3):134-137 
303

304

305

306

307

308

309

310

311

312

Tanaka M (2003) Visual preference by chimpanzees (Pan troglodytes) for photos of primates measured by a free choice-order task: implication for influence of social experience. Primates; journal of primatology 44 (2):157-165. doi:10.1007/s10329-002-0022-8

Tomonaga M (2007) Is chimpanzee (Pan troglodytes) spatial attention reflexively triggered by gaze cue? Journal of comparative psychology 121 (2):156-170. doi:10.1037/0735-7036.121.2.156

Tomonaga M (2008) Relative numerosity discrimination by chimpanzees (Pan troglodytes): evidence for approximate numerical representations. Animal cognition 11 (1):43-57. doi:10.1007/s10071-007-0089-0

Tomonaga M, Imura T (2009) Faces capture the visuospatial attention of chimpanzees (Pan troglodytes): evidence from a cueing experiment. Frontiers in zoology 6:14. doi:10.1186/1742-9994-6-14

Tomonaga M, Imura T (2010) Pacman in the sky with shadows: the effect of cast shadows on the perceptual completion of occluded figures by chimpanzees and humans. Behavioral and brain functions : BBF 6:38. doi:10.1186/1744-9081-6-38

Tucker A, Miyake H, Omura T, Tsuji M, Ukita T, Nishihara K, Oi S (2006) Huge arachnoid cyst of the occipital cerebral convexity. Neurologia medico-chirurgica 46 (7):361-365

Ushitani T, Imura T, Tomonaga M (2010) Object-based attention in chimpanzees (Pan troglodytes). Vision research 50 (6):577-584. doi:10.1016/j.visres.2010.01.003

Vernau KM, Kortz GD, Koblik PD, LeCouteur RA, Bailey CS, Pedroia V (1997) Magnetic resonance imaging and computed tomography characteristics of intracranial intra-arachnoid cysts in 6 dogs. Veterinary radiology \& ultrasound : the official journal of the American College of Veterinary Radiology and the International Veterinary Radiology Association 38 (3):171-176 
327 Wyss-Fluehmann G, Konar M, Jaggy A, Vandevelde M, Oevermann A (2008) Cerebellar ependymal cyst in a dog. Veterinary pathology 45 (6):910-913.

329 doi:10.1354/vp.45-6-910

330 Yamamoto S, Humle T, Tanaka M (2009) Chimpanzees help each other upon request. PloS 331 one 4 (10):e7416. doi:10.1371/journal.pone.0007416

332 Yamamoto S, Tanaka M (2009) Do chimpanzees (Pan troglodytes) spontaneously take turns 333 in a reciprocal cooperation task? Journal of comparative psychology 123 (3):242-249. doi:10.1037/a0015838 
Table 1. Cognitive studies in which Pendesa participated.

* The age of the chimpanzee at which she participated the task. (-) indicates that the age is not stated in the article.

\begin{tabular}{|c|c|c|c|c|c|}
\hline $\begin{array}{l}\text { Task } \\
\text { category }\end{array}$ & Task & Topic & Age* & $\begin{array}{l}\text { Number of } \\
\text { subjects }\end{array}$ & Reference \\
\hline Vision & Concurrent discrimination & Self-monitoring of action & - & 6 & Kaneko and Tomonaga 2012 \\
\hline Vision & Odd item search & Emergent feature & 29 & 3 & Goto et al. 2012 \\
\hline Vision & Concurrent discrimination & Agency judgment & - & 3 & Kaneko and Tomonaga 2011 \\
\hline Vision & Odd item search & Perceptual completion & - & 6 & Tomonaga and Imura 2010 \\
\hline Vision & Pre-cue task & Object based attention & - & 2 & Ushitani et al. 2010 \\
\hline Vision & Pre-cue task & Cueing effect of human pointing & - & 2 & Tomonaga and Imura 2009 \\
\hline Social & Token insertion task & Reciprocal cooperation & 29 & 4 & Yamamoto and Tanaka 2009 \\
\hline Social & Tool transfer task & Helping behaviour & - & 9 & Yamamoto et al. 2009 \\
\hline Vision & Matching to sample & Metacontrast and back/forward masking & 28 & 2 & Matsuno and Tomonaga 2008 \\
\hline Vision & Concurrent discrimination & Relative numerosity discrimination & - & 2 & Tomonaga 2008 \\
\hline Vision & Concurrent discrimination & Pictorial depth cue perception & 24 & 3 & Imura et al. 2008 \\
\hline Vision & Matching to sample & Dominant perception of concave shape & 28 & 5 & Matsuno and Tomonaga 2007 \\
\hline Vision & Pre-cue task & Gaze cueing effect & - & 2 & Tomonaga 2007 \\
\hline Social & Free viewing & Mirror self-recognition & 21 & 10 & Hirata 2007 \\
\hline Vision & Odd item search & Grouping of moving/stationary object & 27 & 3 & Matsuno and Tomonaga 2006 \\
\hline Vision & Matching to sample & Color classification & 23 & 2 & Matsuno et al. 2004 \\
\hline Social & Free viewing & Contagious yawning & 26 & 6 & Anderson et al. 2004 \\
\hline Ecology & Leaf swallowing & Self-medicative behavior & - & 11 & Huffman and Hirata 2004 \\
\hline Motor & Hand drawing & Improvement of manual movement & 15 & 2 & Iversen and Matsuzawa 2003 \\
\hline Vision & Free choice task & Visual preference of photo & 23 & 5 & Tanaka 2003 \\
\hline Social & Open field food detection & Tactical Deception & 20 & 5 & Hirata and Matsuzawa 2001 \\
\hline Social & Toke exchange task & Use of token & - & 3 & Sousa and Matsuzawa 2001 \\
\hline Vision & Object choice task & Recognition of human-given cue & 18 & 2 & Itakura and Tanaka 1998 \\
\hline Motor & Hand drawing & Model guided hand drawing & 15 & 2 & Iversen and Matsuzawa 1997 \\
\hline Motor & Hand drawing & Visually guided hand drawing & 15 & 2 & Iversen and Matsuzawa 1996 \\
\hline Auditory & Go/NoGo & Auditory function & 7 & 2 & Kojima 1990 \\
\hline Auditory & Go/NoGo & Consonant perception & 9 & 2 & Kojima et al. 1989 \\
\hline Auditory & Go/NoGo & Vowel perception & 9 & 3 & Kojima and Kiritani 1989 \\
\hline
\end{tabular}




\section{Figure legends}

Fig. 1

T1-weighted coronal (A), axial (B) and sagittal (C) MRI showing a right occipital arachnoid cyst with a total volume of $2.8 \mathrm{~cm}^{3}$ (contoured area)

(D) 3D reconstructed CT images of the skull. The thickness of the mandible was

asymmetrical (yellow bar), whereas the temporomandibular joints (arrowhead) and dentition were symmetrical. 

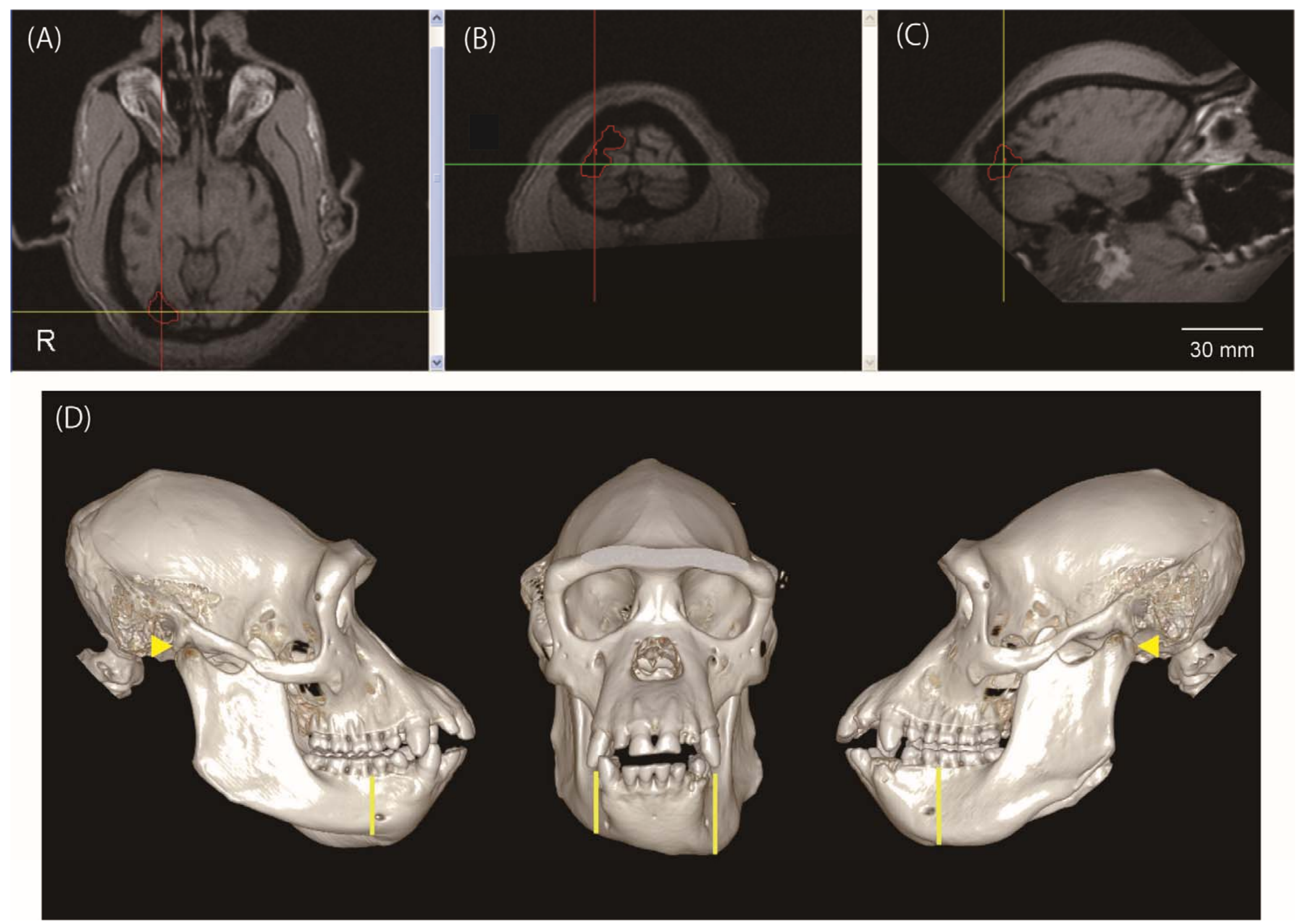